\title{
Epidemiological characteristics and post- exposure prophylaxis of human rabies in Chongqing, China, 2007-2016
}

Li Qi ${ }^{1,2+}$, Kun Su ${ }^{1,3 \dagger}$, Tao Shen ${ }^{2}$, Wenge Tang ${ }^{1}$, Bangzhong Xiao ${ }^{1}$, Jiang Long ${ }^{1}$, Han Zhao ${ }^{1}$, Xi Chen ${ }^{1}$, Yu Xia ${ }^{1}$, Yu Xiong ${ }^{1}$, Dayong Xiao ${ }^{1}$, Liangui Feng ${ }^{4^{*+}}$ and Qin $\mathrm{Li}^{1^{*+}}$

\begin{abstract}
Background: According to the global framework of eliminating human rabies, China is responding to achieve the target of zero human death from dog-mediated rabies by 2030. Chongqing is the largest municipality directly under central government in China. We described the epidemiological characteristics and post-exposure prophylaxis (PEP) of human rabies in this area, in order to provide a reliable epidemiology basis for further control and prevention of human rabies.

Methods: The most updated epidemiological data for human rabies cases from 2007 to 2016 in Chongqing were collected from the National Disease Reporting Information System. A standardized questionnaire was applied to the human rabies cases or family members of cases as proxy to investigate the PEP situation.

Results: A total of 809 fatal human rabies cases were reported in Chongqing from 2007 to 2016. There was a trend of gradual annual decline about number of cases from 2007 to 2013, followed by stable levels until 2016. Rabies was mostly reported in summer and autumn; a majority of cases were noted in farmers (71.8\%), especially in males (65.3\%). The cases aged 35-74 and 5-14 years old accounted for $83.8 \%$ of all the cases. We collected information of 548 human rabies cases' rabies exposure and PEP situation. Of those, $95.8 \%$ of human rabies cases were victims of dog bites or scratch, and $53.3 \%$ of these dogs were identified as stray dogs. Only $4.0 \%$ of the domestic dogs were reported to have been vaccinated previously. After exposure, $87.8 \%$ of the 548 human rabies cases did not seek any medical services. Further investigation showed that none of the 548 cases received timely and properly standardized PEP.

Conclusion: Human rabies remains a major public health problem in Chongqing, China. Dogs are the main reservoir and source of human rabies infection. Unsuccessful control of canine rabies and inadequate PEP of cases might be the main factors leading to the serious human rabies epidemic in this area. An integrated "One Health" approach should be encouraged and strengthened in this area; with combined effort it would be possible to achieve the elimination of human rabies in the expected date.
\end{abstract}

Keywords: Human rabies, Post-exposure prophylaxis, Epidemiology

\footnotetext{
*Correspondence: cqlianguifeng@126.com; qili19812012@126.com

'Equal contributors

${ }^{4}$ Chongqing Medical and Pharmaceutical College, Chongqing, China

${ }^{1}$ Chongqing Municipal Center for Disease Control and Prevention, No.8,

Changjiang 2nd Road, Yuzhong District, Chongqing, China

Full list of author information is available at the end of the article
} 


\section{Background}

Rabies is a neglected zoonosis infectious disease and the most lethal infectious disease with fatality rate in humans approaching $100 \%$ [1]. The number of human rabies deaths was estimated to be 61,000 globally [2], corresponding to over 3.7 million disability-adjusted life-years and 8.6 billion USD economic losses every year [3]. China is one of the countries that experience the most serious impact from this disease, with human rabies cases ranking second in the world, after India [4, 5]. The 2015 Chinese yearbook of health statistics showed that rabies death ranked the third in the list of 39 notifiable infectious diseases, following AIDS and tuberculosis [6]. The disease was severely underreported because most victims died at home, which led to an insufficient prioritization of rabies prevention in public health agendas [7]. Chongqing is the largest municipality under direct control of the national government, located in the Southwestern region of China, sharing borders with Hunan Province and Guizhou Province, which are two of the most seriously affected provinces by human rabies in China [8].

According to the global framework of eliminating rabies, China is responding to the target of zero human rabies death by 2030 by scaling up their response to consign rabies to the history books [1]. Epidemiological surveys and phylogenetic analyses indicate elimination of rabies in wild animals and stray dogs are important for control of human rabies in China [4]. Further understanding on these issues would assist the development of measures such as better vaccines for control of human rabies $[4,9]$.

It is necessary to understand the local epidemiological characteristics of human rabies for providing a reliable epidemiology basis for further control and prevention of human rabies in Chongqing. Therefore, we carried out an epidemiological analysis of the human rabies surveillance data and the information on the post-exposure prophylaxis (PEP) of human rabies cases from 2007 to 2016, in order to improve the public health management strategies in Chongqing.

\section{Methods}

\section{Information sources}

The epidemiological data of human rabies in Chongqing from January 2007 to December 2016 were retrieved from the surveillance database of the "National Disease Reporting Information System" (NDRIS) of the Chinese Center for Disease Control and Prevention (CDC). According to the law of the People's Republic of China on Prevention and Treatment of Infectious Diseases, all human rabies cases should be reported to NDRIS within $24 \mathrm{~h}$ after diagnosis. Human rabies is diagnosed according to the National Diagnostic Criteria for Rabies (WS 281-2008).
To investigate the situation of rabies exposure and the PEP of human rabies cases, a standardized questionnaire was administered face-to-face to 548 cases or their family members by trained investigators after informed and consent. The questionnaire covered four parts: (a) the cases' demographic profile (name, gender, age, occupation); (b) exposure characteristics (date of event, site of lesion, the animal vector, the category of exposure); (c) PEP (wound washing, vaccination and/or immunoglobulin administration); and (d) clinical manifestation. The categories of exposure were classified according to the World Health Organization's (WHO) guideline [10].

\section{Statistical analysis}

Initial data were entered into EpiData software 3.1 and analyzed. Descriptive analyses were performed and presented in percentage or median and interquartile range to describe the demographic characteristics of the cases. The incubation time was assessed by Kruskal-Wallis $\mathrm{H}$ test or Kolmogorov-Smirnov $\mathrm{Z}$ test wherever appropriate. $P<0.05$ was considered to be statistically significant.

\section{Results \\ Epidemiological characteristics}

According to NDRIS, a total of 809 human rabies cases were reported and died in Chongqing from 2007 to 2016 , the annual average incidence of the disease was 0.3 per 100,000 inhabitants per year. The annual incidences of human rabies showed a decreasing trend from 2008 to 2013, and then remained stable from 2013 to 2016. The annual number of cases and incidence rates for human rabies are summarized in Fig. 1.

Human rabies cases were reported throughout the year. However, $64.2 \%$ (519/809) of the cases occurred in summer or autumn seasons (from May to October) and the numbers reached peak in October, accounting for

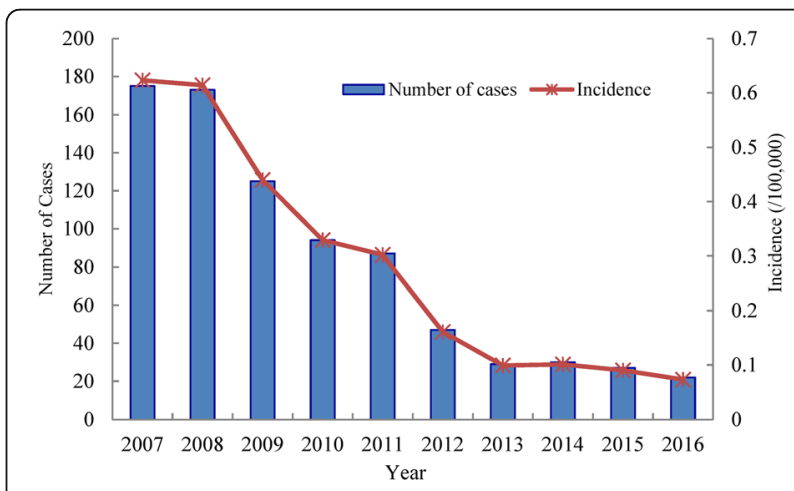

Fig. 1 The annual number of cases and incidence rate for human rabies in Chongqing, China, 2007-2016. The blue columns indicate the number of human rabies cases and the red line indicates the incidence rate of human rabies cases 
$13.5 \%$ of the total cases. The monthly distribution of human rabies cases are shown in Fig. 2 (see Additional file 1: Supporting file 1 for more details).

The age of human rabies cases ranged from 1 to 93, with a median of 53 years old. The peak ages of the cases fell in the 5-14 and 35-74 age groups, with 113 and 565 cases, respectively, which accounted for nearly $83.8 \%$ of the total cases. The male to female ratio was 1.9:1. The highest number of cases was found in farmers, which accounted for $71.8 \%$ of the total cases, followed by students or kindergarten-age children (15.4\%). Overall, these two populations jointly accounted for $87.2 \%$ of the total cases. Demographic characteristics of human rabies cases from 2007 to 2016 in Chongqing were listed in Table 1.

The top four districts or counties with largest numbers of human rabies in Chongqing were Wanzhou (54 cases), Hechuan (52 cases), Tongnan (49 cases) and Fuling (47 cases), and all of them are rural areas.

\section{Exposure and incubation}

Exposure and PEP information were collected from 548 $(67.7 \%)$ of all the reported rabies cases from 2007 to 2016. Of them, 525 (95.8\%) cases had a history of dog bite or scratch and another $20(3.6 \%)$ were attributed to cats (Table 2). Of the animal vectors, 292 (53.3\%) were identified as stray animals and $253(46.2 \%)$ were domestic dogs (belonging to the family of the case or a neighbor). Only $4.0 \%(10 / 253)$ of the domestic dogs had received rabies vaccine.

According to the classification of contact with animals issued by WHO, 137 (25.0\%) cases were classified into category II exposure (i.e., nibbling of uncovered skin, or minor abrasions without bleeding) and 411 (75.0\%) were classified into category III exposure (i.e., transdermal bites or scratches, or mucous membrane contamination). The major wounds were occurred on the upper limbs (45.8\%), followed by the lower limbs (31.6\%), the

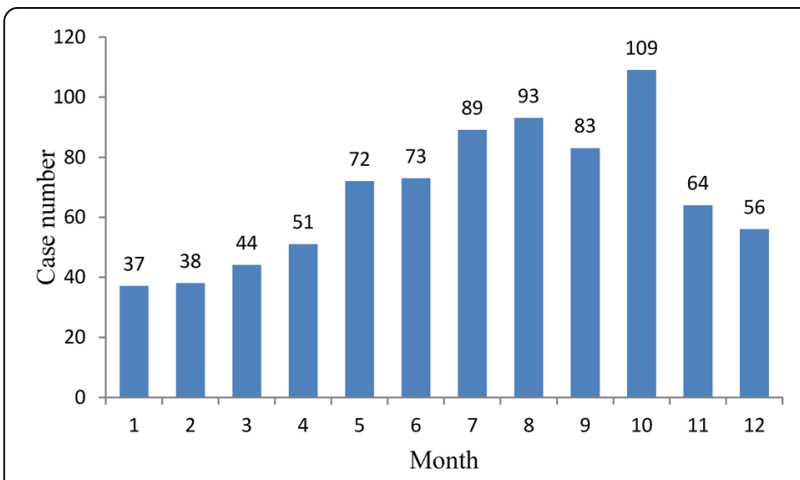

Fig. 2 The human rabies cases by month in Chongqing, China, 2007-2016. The blue columns indicate the number of human rabies cases per month
Table 1 Characteristics of human rabies cases in Chongqing, China, 2007-2016

\begin{tabular}{|c|c|c|}
\hline Characteristics & $\begin{array}{l}\text { Number of human } \\
\text { rabies cases }\end{array}$ & Proportion (\%) \\
\hline \multicolumn{3}{|l|}{ Age group } \\
\hline 0 to 4 years & 33 & 4.1 \\
\hline 5 to 9 years & 57 & 7.1 \\
\hline 10 to 14 years & 56 & 6.9 \\
\hline 15 to 24 years & 27 & 3.3 \\
\hline 25 to 34 years & 27 & 3.3 \\
\hline 35 to 44 years & 99 & 12.2 \\
\hline 45 to 54 years & 122 & 15.1 \\
\hline 55 to 64 years & 230 & 28.4 \\
\hline 65 to 74 years & 114 & 14.1 \\
\hline 75 to 93 years & 44 & 5.5 \\
\hline \multicolumn{3}{|l|}{ Gender } \\
\hline Male & 528 & 65.3 \\
\hline Female & 281 & 34.7 \\
\hline \multicolumn{3}{|l|}{ Occupation } \\
\hline Farmer & 581 & 71.8 \\
\hline $\begin{array}{l}\text { Students or Kindergarten-age } \\
\text { children }^{\mathrm{a}}\end{array}$ & 125 & 15.5 \\
\hline Scattered children ${ }^{\mathrm{b}}$ & 31 & 3.8 \\
\hline Housewife or Retiree & 17 & 2.1 \\
\hline Worker & 14 & 1.7 \\
\hline Medical staff & 2 & 0.3 \\
\hline Others & 39 & 4.8 \\
\hline
\end{tabular}

${ }^{a}$ Students or Kindergarten-age children refer to those students studied in kindergarten, primary school, middle school or university

${ }^{\mathrm{b}} \mathrm{S}$ cattered children refer to children aged less than 3 years old

remaining were on the head/ neck (17.3\%) and trunk (5.3\%).

According to the available data on the incubation period of 517 cases, the median was 54 days (ranged from 2 to 1476 days). The majority of cases occurred within 30 incubation days $(29.0 \%)$, followed by the $30-60$ days $(25.3 \%)$. The incubation period of $72.7 \%$ cases was less than 3 months, while 13 cases (2.5\%) had an incubation period over 1 year. Six cases $(1.2 \%)$ had a short incubation period of less than 1 week after recognized exposure, while the longest reported incubation period in this study was 4 years, following a dog bite vaguely recalled by the case's relative.

The incubation period was significantly shorter in cases with exposure sites on the head/neck $($ median $=22$ ) than cases with a wound on trunk, upper or lower limbs $(p<0.01)$ (Table 3$)$. Similarly, the incubation period was significantly shorter in cases with III exposure than in cases with II exposure $(p=0.031)$. 
Table 2 Exposure characteristics of human rabies cases in Chongqing, 2007-2016

\begin{tabular}{|c|c|c|}
\hline Exposure characteristics & $\begin{array}{l}\text { No. of human } \\
\text { rabies cases }\end{array}$ & Proportion (\%) \\
\hline \multicolumn{3}{|l|}{ Animal vector } \\
\hline Canine & 525 & 95.8 \\
\hline Cat & 20 & 3.7 \\
\hline Wild animal & 3 & 0.5 \\
\hline \multicolumn{3}{|l|}{ Animal source } \\
\hline Stray dogs or cats & 292 & 53.3 \\
\hline Domestic dogs & 253 & 46.2 \\
\hline Wild & 3 & 0.5 \\
\hline \multicolumn{3}{|l|}{ Exposure reasons } \\
\hline Active attack by the vector & 404 & 73.7 \\
\hline Defensive attack by the vector & 55 & 10.0 \\
\hline Playing with the vector & 69 & 12.6 \\
\hline Other & 20 & 3.7 \\
\hline \multicolumn{3}{|l|}{ Exposure level $^{\mathrm{a}}$} \\
\hline III & 411 & 75.0 \\
\hline$\|$ & 137 & 25.0 \\
\hline \multicolumn{3}{|l|}{ Exposure site } \\
\hline Upper limb & 251 & 45.8 \\
\hline Lower limb & 173 & 31.6 \\
\hline Head/neck & 95 & 17.3 \\
\hline Trunk & 29 & 5.3 \\
\hline \multicolumn{3}{|l|}{ Incubation period } \\
\hline$\leq 30$ days & 150 & 29.0 \\
\hline 30-60 days & 131 & 25.3 \\
\hline $61-90$ days & 95 & 18.4 \\
\hline $91-120$ days & 53 & 10.3 \\
\hline $121-150$ days & 20 & 3.9 \\
\hline $151-180$ days & 15 & 2.9 \\
\hline 181-365 days & 40 & 7.7 \\
\hline$>1$ year & 13 & 2.5 \\
\hline
\end{tabular}

${ }^{a}$ Category II exposure: nibbling of uncovered skin, minor abrasions or abrasions without bleeding. Category III exposure: single or multiple transdermal bites or scratches, licks on broken skin, contamination of mucous membrane with saliva from licks

\section{Use of rabies post exposure prophylaxis (PEP)}

Of the 548 cases with available PEP information, 290 cases $(52.9 \%)$ had no wound treatment post exposure at all. Another 191 cases (34.9\%) treated the wound by themselves, and only 67 cases $(12.2 \%)$ went to the hospital for medical treatment (Table 4).

With regard to the post-exposure vaccination, 69 cases (12.6\%) received one to four doses of rabies vaccine, only 8 cases $(1.5 \%)$ completed the full regimen of 5 doses, and the remaining cases did not receive any dose. Of the
Table 3 The incubation periods among different exposure levels and sites of the human rabies cases in Chongqing, China, 2007-2016

\begin{tabular}{lll}
\hline Variables & Median (days) & Interquartile range (days) \\
\hline Exposure site & & \\
Head/neck & 22 & 14,50 \\
Trunk & 43 & $14.5,92$ \\
Upper limb & 55 & 31,91 \\
Lower limb & 75 & 44,145 \\
Exposure level & & \\
II & 68 & 35,115 \\
III & 50 & 25,89 \\
\hline
\end{tabular}

8 cases who received the full regimen of 5 doses, 7 cases were category III exposure but did not received rabies immunoglobulin (RIG) which was recommended in WHO guidelines, and 6 cases did not receive the first shot of rabies vaccination on time. In summary, none of the 548 cases investigated in this study received adequate PEP according to the WHO guidelines. A total of 14 cases comprised of 13 with category III exposure and 1 with category II exposure received RIG.

\section{Discussion}

A total of 809 human rabies cases were reported in Chongqing, China, from 2007 to 2016, all of them were dead. The number of human rabies cases gradually

Table 4 Post-exposure prophylaxis of human rabies cases in Chongqing, 2007-2016

\begin{tabular}{|c|c|c|}
\hline Variables & $\begin{array}{l}\text { Number of human } \\
\text { rabies cases }\end{array}$ & Proportion (\%) \\
\hline \multicolumn{3}{|l|}{ Wound treatment } \\
\hline Untreated & 290 & 52.9 \\
\hline Self-treated & 191 & 34.9 \\
\hline Treatment in medical institution & 67 & 12.2 \\
\hline \multicolumn{3}{|l|}{ Active immunization } \\
\hline No & 471 & 85.9 \\
\hline Incomplete full regimen & 69 & 12.6 \\
\hline Complete full regimen & 8 & 1.5 \\
\hline \multicolumn{3}{|l|}{ Passive immunization } \\
\hline Yes & $14^{\mathrm{a}}$ & 2.6 \\
\hline No & 534 & 97.4 \\
\hline \multicolumn{3}{|l|}{ Treatment time for PEP (days) } \\
\hline 0 & 241 & 94.9 \\
\hline $1-3$ & 6 & 2.3 \\
\hline$\geq 4$ & 7 & 2.8 \\
\hline
\end{tabular}

${ }^{\text {a }}$ The total 14 cases that received RIG comprised of 13 cases of category III exposure and 1 case of category II exposure

${ }^{b}$ The treatment time for PEP refers to the interval between potential exposure and receive PEP 
decreased from 2007 to 2013, which is in consistence with the epidemic trend of human rabies in China. This phenomenon may be due to rabies control being emphasized and strengthened by central government in China after 2007. The Treatment Guidelines for Rabies Postexposure Prophylaxis of Humans were updated and applied then, which may attribute to the decline of human rabies cases. However, the human rabies cases remained stable from 2013 to 2016 (22-30 cases per year) in Chongqing, which clearly demonstrated that human rabies constituted a real public health issue in this area.

The major proportion of human rabies cases occurred in summer or autumn seasons, when more frequent outdoor activities with less cloth wearing increased the chance of rabid dog contact and exposure. Geographical distribution of human rabies indicated that most of the cases were in rural areas. The proportion of farmers in the cases was significantly higher than that in the total population [11] (see Additional file 1: Supporting file 2 for details). This phenomenon might be related to the chance of exposure, delay in obtaining PEP after potential rabies exposure (see Additional file 1: Supporting file 3 for details), lower proportion of cases that sought medical treatment (see Additional file 1: Supporting file 4 for details), and the long distance to health centers to receive PEP in rural areas [12].

It is noteworthy that besides adults aged 35-74 years old, children aged 5-14 years old also constituted the major cases. For fear of being scolded, children may not tell their guardians after bitten by animals; therefore they might not receive any treatment. Moreover, due to their height, they were more likely to have exposures on head/neck compared with adults [13] (see Additional file 1: Supporting file 5 for details). Similar to other studies [14-18], males were more frequently infected than females and the proportion of males in cases was significantly higher than that in the total population (see Additional file 1: Supporting file 2 for details); this phenomenon was possibly related to occupational or behavioral factors that making them more frequently contact with the animal vectors. Therefore, children and rural residents especially males aged $35-70$ years could be the priority targets for rabies control and prevention in Chongqing, people should be more vigilant during summer and autumn periods to avoid contacting with animals.

The median incubation period was 54 days for human rabies cases in Chongqing, which consisted with the incubation period stated by the WHO (1-3 months). Similar to the results of other studies $[19,20]$, the incubation period was different for cases with different exposure sites and levels. Cases with a wound on the head/neck had the shortest incubation period compared to those with wounds on other sites; the incubation period of cases with category III exposure was significantly shorter than that of cases with category II exposure. The 6 special cases with incubation period less than 1 week were multiple transdermal bites on head/neck and didn't receive any PEP. Those cases with an incubation period over 1 year suffered minor abrasions or abrasions without bleeding and were regarded as category II exposure. It is worth noting that $25 \%$ of the human rabies cases had a category II exposure in our study, which emphasized the importance of timely and appropriate PEP for exposure without bleeding.

Rabies is almost $100 \%$ fatal once clinical symptoms appear. Fortunately, it can be prevented by giving timely and appropriate PEP [21-23]. The WHO recommends immediate and thorough wound washing for a minimum of $15 \mathrm{~min}$ with soap and flowing water, full regimen of rabies vaccine and RIG for cases with category III exposure, which is considered as severe exposure. However, few cases sought medical treatment in our study, and none of them received adequate PEP according to the WHO guidelines. These results indicated the public's ignorance of human rabies in Chongqing, which was more serious than in other areas $[15,19,24]$. The reasons that cases did not receive recommended PEP after exposure were not investigated in our study, it may be related to several factors, such as the prohibitive costs of vaccine and RIG, the lack of awareness about necessity of PEP and inadequate access to vaccine and RIG in primary health services especially in rural areas [25] (see Additional file 1: Supporting file 6 for details). Therefore, appropriate actions should be implemented and strengthened including (1) increase subsidies for rabies medication by expanding the scope of medical insurance for rabies PEP, just as some high-incidence human rabies provinces in China like Hunan and Guangdong, which have included rabies PEP expenditures into the new rural cooperative medical reimbursement coverage, (2) educate the public on the need of rapid treatment post exposure [26] and use education approaches such as short-messageservice or WeChat, which might be useful tools for health promotion [27], (3) educate public health workers to evaluate and identify potential exposures to rabies and provide adequate PEP [28], (4) establish "animal bite treatment centers" in rural areas for better treatment and surveillance of rabies and animal bites in Chongqing.

Dogs play an important role in the transmission of human rabies infections in Chongqing, which is similar to other countries in Asia, Latin America, and Africa $[12,16,19,29,30]$. The exposure investigation in our study revealed that only $4.0 \%$ of the domestic dogs related to the cases were reported that have been 
vaccinated previously, which is far lower than the threshold of $70 \%$ vaccination coverage for efficient rabies control. Stray dogs were the principal animal responsible for the transmission of rabies in Chongqing. Therefore, mass implementation of canine vaccination of domestic dogs and effective measures to control stray dogs were the most logical solutions to reduce the burden of human rabies in Chongqing, rather than solely focusing on human PEP [31, 32].

Successful experiences of the elimination of human rabies in regions, such as Europe, Japan, Caribbean and Latin American provide a promising method for Chongqing to develop a more effective approach for controlling human rabies [33-35]. Such programs include implementing mass dog vaccination and management, canine rabies outbreak surveillance, health education to the general public especially high risk populations, implementing routine surveillance of rabies exposure in schools, and training the medical personnel to provide appropriate PEP for any potential exposure; these focused on the concept of "One Health" and required interdisciplinary collaboration from the public health and veterinary services, ministries of education, local authorities and non-governmental organizations [4, 36-39].

To our knowledge, this is the most comprehensive study showing the situation of human rabies in Chongqing, China. The data obtained from NDRIS may not well capture all the human rabies cases during this period in Chongqing because of the following reasons: some people in remote rural areas may not have easy access to medical treatment and clinical diagnosis, which lead to underestimated rabies incidence due to the confusion with other neurological infections $[40,41]$. Thus, it is possible that the number of human rabies cases in this study were underreported. Furthermore, some limitations of this study merit noting, including the lack of laboratory testing to confirm rabies, recall bias (particular for cases that involved a long incubation period or unconscious cases whose information recollections depended almost entirely on their family members or relatives) and incomplete information of PEP for several cases. Despite these limitations, this study provides useful information on the profile of human rabies in Chongqing, which could contribute to the control and prevention strategies for human rabies in this area.

\section{Conclusions}

Human rabies remains a major public health problem in Chongqing, China. Unsuccessful control of canine rabies and inadequate PEP of cases might be the main factors leading to the serious human rabies epidemic in this area. It is crucial to implement a series of programs based on the concept of "One Health", including implementing mass rabies vaccination, strict control of stray dogs, education of the public on the risk and prevention of rabies, implementing routine surveillance of rabies exposure in schools, and training medical personnel to provide appropriate PEP. As such, the elimination of human rabies in this area in the end will then be possible.

\section{Additional file}

Additional file 1: Tables S1. The monthly distribution of human rabies cases in Chongqing from 2007 to 2016. Table S2. Comparison of the population distribution of human cases with that of the local population in Chongqing. Table S3. The interval of exposure and treatment time for PEP. Table S4. The proportion of cases that sought medical treatment. Table S5. The proportion of cases with exposure on head/neck. Table S6. PEP ambulant clinic conditions in Chongqing, 2016. (XLSX 20 kb)

\section{Abbreviations}

AIDS: Acquired immunodeficiency syndrome; CDC: Center for disease control and prevention; NDRIS: National disease reporting information system; PEP: Post-exposure prophylaxis; RIG: Rabies immunoglobulin; USD: United States dollar; WHO: World Health Organization

\section{Acknowledgments}

The authors would like to acknowledge the contribution of the survey team members and thank for JingXin Li, Qin Liu, Yong Zhao and Ke Ning for their thoughtful suggestions and insights, which have enriched the manuscript and produced a more balanced and better account of the research. We also express our special thanks to all interviewees for their participation and to the Chinese Center for Disease Control and Prevention for their technical assistance.

\section{Funding}

This research was supported by the National Key R\&D Program of China (2017YFC0907300)

\section{Availability of data and materials}

The datasets analyzed during the current study are available from the corresponding author on reasonable request.

\section{Authors' contributions}

LQ, KS, LGF and QL conceived of and designed this study. LQ, KS, QL and TS acquired, analyzed and interpreted the data. LQ, KS, TS and LGF drafted the manuscript and critically revised the manuscript for important intellectual content. LQ, KS, QL, LGF, TS, WGT, BZX, JL, HZ, XC, YX (Xia), YX (Xiong) and DYX have participated sufficiently in the work and agreed to be accountable for all aspects of the work. All authors read and approved the final manuscript.

\section{Ethics approval and consent to participate}

Ethical clearance for this study was granted by the Ethic Committee of Chongqing Center for Disease Control and Prevention. Written informed consent was obtained from the cases or their' families before the detailed information were collected. All data analyzed in this study were anonymized.

\section{Consent for publication}

Not applicable.

Competing interests

The authors declare that they have no competing interests.

\section{Publisher's Note}

Springer Nature remains neutral with regard to jurisdictional claims in published maps and institutional affiliations.

\section{Author details}

${ }^{1}$ Chongqing Municipal Center for Disease Control and Prevention, No.8, Changjiang 2nd Road, Yuzhong District, Chongqing, China. ${ }^{2}$ Chinese Field 
Epidemiology Training Program, Chinese Center for Disease Control and Prevention, Beijing, China. ${ }^{3}$ Department of Military Epidemiology, College of Military Prevention, Third Military Medical University, Chongqing, China. ${ }^{4}$ Chongaing Medical and Pharmaceutical College, Chongqing, China.

\section{Received: 22 May 2017 Accepted: 12 November 2017}

\section{Published online: 03 January 2018}

\section{References}

1. Baghi HB, Bazmani A, Aghazadeh M. The fight against rabies: the Middle East needs to step up its game. Lancet. 2016:388(10054):1880.

2. WHO. WHO expert consultation on rabies: second report. Geneva: World Health Organization; 2013.

3. Hampson K, Coudeville L, Lembo T, Sambo M, Kieffer A, Attlan M, et al. Estimating the global burden of endemic canine rabies. PLoS Negl Trop Dis. 2015;9(4):e0003709.

4. Tan J, Wang R, Ji S, Su S, Zhou J. One health strategies for rabies control in rural areas of china. Lancet Infect Dis. 2017;17(4):365-7.

5. Liu Q, Cao L, Zhu XQ. Major emerging and re-emerging zoonoses in China: a matter of global health and socioeconomic development for 1.3 billion. Int J Infect Dis. 2014;25:65-72.

6. $\mathrm{MOH}$ (2015) Infectious Disease of Mandatory Notification. http://www. phsciencedata.cn/Share/ky_sjml.jsp?id=a8589320-504d-465a-a87223017a8a03a0. Accessed: 21 Feb 2017.

7. Yang GJ, Liu L, Zhu HR, Griffiths SM, Tanner M, Bergquist R, et al. China's sustained drive to eliminate neglected tropical diseases. Lancet Infect Dis. 2014;14(9):881-2.

8. Miao S, Tang Q, Wang DM, Mo ZJ, Guo SH, Hao L, et al. Epidemiological investigations of human rabies in China. BMC Infect Dis. 2009;9:210.

9. He W, Zhang H, Zhang Y, Wang R, Lu S, Ji Y, et al. Codon usage bias in the $\mathrm{N}$ gene of rabies virus. Infect Gene Evol J Mol Epidemiol Evol Gene Infect Dis. 2017:54:458-65.

10. Tang Q. Epidemic situation and related factors analyses of rabies in China. Chin J Epidemiol. 2005;26(3):2.

11. Chongqing statistical yearbook (2016): Available from: http://www.cqtj.gov. cn/tjsj/sjjd/. Accessed 21 Feb 2017.

12. Davlin SL, Vonville HM. Canine rabies vaccination and domestic dog population characteristics in the developing world: a systematic review. Vaccine. 2012;30(24):3492-502.

13. Ponsich A, Goutard F, Sorn S, Tarantola A. A prospective study on the incidence of dog bites and management in a rural Cambodian, rabiesendemic setting. Acta Trop. 2016;160:62-7.

14. Montgomery JP, Zhang Y, Wells EV, Liu Y, Clayton JL, Wang X, et al. Human rabies in Tianjin, China. J Public Health. 2012;34(4):505-11.

15. Li GW, Chen QG, Qu ZY, Xia Y, Lam A, Zhang DM, et al. Epidemiological characteristics of human rabies in Henan province in China from 2005 to 2013. J Venom Anim Toxins Incl Trop Dis. 2015;21:34

16. Susilawathi NM, Darwinata AE, Dwija IB, Budayanti NS, Wirasandhi GA, Subrata K, et al. Epidemiological and clinical features of human rabies cases in Bali 2008-2010. BMC Infect Dis. 2012;12:81.

17. Tenzin DNK, Ward MP. Human rabies post exposure prophylaxis in Bhutan, 2005-2008: trends and risk factors. Vaccine. 2011;29(24):4094-101.

18. Mohtasham AZ, Pourmarzi DR, Razi M. Epidemiology of dog bite, a potential source of rabies in Guilan, north of Iran. Asian Pac J Trop Dis. 2015;5 (suppl 1):S104-8

19. Ren J, Gong Z, Chen E, Lin J, Lv H, Wang W, et al. Human rabies in Zhejiang Province, China. Int J Infect Dis. 2015;38(C):77-82.

20. Yang JY, Tan Y, Zhaojun MO, Jianjun MO, Zhou K. Epidemiology of human rabies in guangxi, china, 1951-2010. Chin J Zoonoses. 2013;29(3):294-9.

21. Dhankhar P, Vaidya SA, Fishbien DB, Meltzer MI. Cost effectiveness of rabies post exposure prophylaxis in the United States. Vaccine. 2008;26(33):4251-5.

22. Takayama N. Rabies: a preventable but incurable disease. J Infect Chemother. 2008;14(1):8-14.

23. Wilde H, Lumlertdacha B, Meslin FX, Ghai S, Hemachudha T. Worldwide rabies deaths prevention-a focus on the current inadequacies in postexposure prophylaxis of animal bite victims. Vaccine. 2016;34(2):187-9.

24. Zhou H, Vong S, Liu K, Li Y, Mu D, Wang L, et al. Human rabies in China, 1960-2014: a descriptive epidemiological study. PLoS Negl Trop Dis. 2016; 10(8):e0004874.
25. Joseph JS, Khan AM, Rajoura OP. Determinants of delay in initiating postexposure prophylaxis for rabies prevention among animal bite cases: hospital based study. Vaccine. 2013;32(1):74-7.

26. 10 facts on rabies. Available from: http://www.who.int/features/factfiles/ rabies/en/. Accessed: 21 Feb 2017.

27. Wu H, Chen J, Zou L, Zheng L, Zhang W, Meng Z, et al. Community-based interventions to enhance knowledge, protective attitudes and behaviors towards canine rabies: results from a health communication intervention study in Guangxi, China. BMC Infect Dis. 2016;16(1):701.

28. Joseph JN, Khan AM, Rajoura OP. Determinants of delay in initiating postexposure prophylaxis for rabies prevention among animal bite cases: hospital based study. Vaccine. 2013;32(1):74-7.

29. Ortiz-Prado E, Ponce-Zea J, Ramirez D, Stewart-Ibarra AM, Armijos L, Yockteng J, et al. Rabies epidemiology and control in Ecuador. Glob J Health Sci. 2015;8(3):113-21.

30. Seimenis A. The rabies situation in the Middle East. Dev Biol. 2008;131:43-53.

31. Weyer J, Szmyd-Potapczuk AV, Blumberg LH, Leman PA, Markotter W, Swanepoel R, et al. Epidemiology of human rabies in South Africa, 1983-2007. Virus Res. 2011;155(1):283-90.

32. Tenzin WK, Ward MP. Human and animal rabies prevention and control cost in Bhutan, 2001-2008: the cost-benefit of dog rabies elimination. Vaccine. 2012:31(1):260-70

33. Vigilato MA, Cosivi O, Knöbl T, Clavijo A, Silva HM. Rabies update for Latin America and the Caribbean. Emerg Infect Dis. 2013:19(4):678-9.

34. Avelino A, Miléia RP, Célio NS, Hugo VU, Mário HT. Humoral immune response in dogs and cats vaccinated against rabies in southeastern Brazil. J Venom Anim Toxins Incl Trop Dis. 2013;19(1):17.

35. Babboni SD, Da CH, Martorelli LD, Kataoka AP, Victoria C, Padovani CR, et al. Kinetics of rabies antibodies as a strategy for canine active immunization. J Venom Anim Toxins Incl Trop Dis. 2014;20(1):1-4.

36. Franka R, Smith TG, Dyer JL, Wu X, Niezgoda M, Rupprecht CE. Current and future tools for global canine rabies elimination. Antivir Res. 2013;100(1):220-5.

37. Blueprint for Rabies Prevention and Control. http://www.rabiesblueprint. com/. Accessed: 21 Feb 2017.

38. Abela-Ridder B, Knopf L, Martin S, Taylor L, Torres G, De Balogh K. 2016: the beginning of the end of rabies? Lancet Glob Health. 2016;4(11):e780-1.

39. Lembo T. The blueprint for rabies prevention and control: a novel operational toolkit for rabies elimination. PLoS Negl Trop Dis. 2012;6(2):e1388.

40. Mallewa M, Fooks AR, Banda D, Chikungwa P, Mankhambo L, Molyneux E, et al. Rabies encephalitis in malaria-endemic area, Malawi, Africa. Emerg Infect Dis. 2007;13(1):136-9.

41. Kieny MP, Hampson K, Dobson A, Kaare M, Dushoff J, Magoto M, et al. Rabies exposures, post-exposure prophylaxis and deaths in a region of endemic canine rabies. PLoS Negl Trop Dis. 2008;2(11):e339.

\section{Submit your next manuscript to BioMed Central and we will help you at every step:}

- We accept pre-submission inquiries

- Our selector tool helps you to find the most relevant journal

- We provide round the clock customer support

- Convenient online submission

- Thorough peer review

- Inclusion in PubMed and all major indexing services

- Maximum visibility for your research

Submit your manuscript at www.biomedcentral.com/submit
) Biomed Central 